\section{Case Reports in Oncology}

\title{
Intracardiac Extension of Wilms Tumor: A Case of a 2.5-Year-Old Girl Presenting with Upper Venous Congestion Caused by Tumor Growth into the Right Cardiac Ventricle
}

Roland Imle Georgi Tosev $^{\mathrm{b}} \quad$ Wolfgang Behnisch $^{\mathrm{a}} \quad$ Jens-Peter Schenk ${ }^{\mathrm{c}}$ Helmut Rauch $^{d}$ Andreas Mueller ${ }^{\mathrm{e}}$ Matthias Gorenflo ${ }^{\mathrm{e}}$ Tsvetomir Loukanov $^{f}$ Andreas Kulozik ${ }^{a}$ Joanne Nyarangi-Dix ${ }^{b}$

aDepartment of Pediatric Oncology, Hematology and Immunology, University of Heidelberg, Heidelberg, Germany; ${ }^{b}$ Department of Urology, University of Heidelberg, Heidelberg, Germany; 'Division of Pediatric Radiology, Clinic of Diagnostic and Interventional Radiology, University of Heidelberg, Heidelberg, Germany; dDepartment of Anesthesiology, University of Heidelberg, Heidelberg, Germany; ${ }^{e}$ Department of Pediatric Cardiology, University of Heidelberg, Heidelberg, Germany; ${ }^{\text {fDepartment }}$ of Cardiac Surgery, Division of Pediatric Cardiac Surgery, Heidelberg, Germany

\section{Keywords}

Wilms Tumor · Intravascular tumor extent · Intracardiac tumor extent · Cardiopulmonary bypass - Neoadjuvant chemotherapy

\begin{abstract}
While Wilms tumors (WT) typically present solely with an abdominally palpable mass, rare cases exhibiting vascular tumor growth can also present with circulatory problems. Here, we report the case of a 2.5-year-old girl presenting with upper venous congestion and arterial hypertension as the primary symptoms of intraventricular tumor growth exhibiting remarkable tubular and perfused morphology. Clinical situation stabilized after initiation of neoadjuvant
\end{abstract}


chemotherapy (NAC) with actinomycin D and vincristine, followed by surgical resection via laparotomy and sternotomy supported by cardiopulmonary bypass and deep hypothermia. Our results highlight the previously reported feasibility of this approach, even in primarily unstable patients.

\section{Introduction}

While WT is the most common kidney malignancy in children with a yearly incidence of about 1 per 100,000, intravascular manifestation is a rarity, occurring in only about 3 to $10 \%$ of WT cases [1]. About a third of these reach the right cardiac atrium and only singular cases have been reported to reach beyond the tricuspid valve into the right cardiac ventricle $[2,3$, $4,5]$, making it even rarer than bilateral WT, which occurs in $4-13 \%$ of patients [6]. The rarity of this condition is especially underlined by the SIOP93-01 and SIOP2001 studies (Societe internationale D'oncologie pediatrique): Of a total number of 1,151 patients with 33 exhibiting intravascular WT growth, not a single case extended to the right cardiac ventricle [1]. Overall 5-year survival rates in WT patients are about $90 \%$, including about $70 \%$ for metastatic disease [7]. WT with intravascular extent has been shown to primarily metastasize in only about $50 \%$ of cases and nearly $80 \%$ of vascularly extended WTs show the favorable intermediate risk histology while only $15 \%$ fall into the high risk group [1].

A staging system for intravascular WT growth was introduced 1994 by Daum et al who adopted previous scores from renal cell carcinoma (stages 1-4: 1. renal vein; 2 . VCI [vena cava inferior] beneath liver veins; 3 . VCI extension to liver veins 4 . intraatrial extension) and suggested application of cardiopulmonary bypass and deep hypothermia for stages 3 and 4 [8]. Abdullah et al later added stage 5 for intraventricular extension [2].

\section{Case Presentation}

A 2.5-year old previously healthy and appropriately developed girl presented with a 1week history of fatigue. One day prior to consultation of the family pediatrician she developed facial and leg edema, vomited once and was transferred to the pediatric emergency unit with suspected diagnosis of nephrotic syndrome. Urine analysis showed albuminuria of 1,624.0 $\mathrm{mg} / \mathrm{L}(\mathrm{n}<20)$. Upon initial physical examination she exhibited facial and leg edema, arterial hypertension with blood pressure of 152/112 $\mathrm{mm} \mathrm{Hg}$ (percentile >99\%), heart rates of about 160/min and a 3/6 systolic murmur with maximum intensity over Erb's point. There was no clearly palpable abdominal mass and initial laboratory analysis only showed mildly elevated LDH at 482 IU/L ( $<364)$. Later determined NT-pro-BNP was highly elevated at 5,046 ng/L ( $\mathrm{n}<125)$, NSE was at $171.4 \mu \mathrm{g} / \mathrm{L}(\mathrm{n} \leq 22)$, Vanillyl mandelic acid and homovanillic acid in urine analysis and broad screening for infectious diseases were within normal limits.

Abdominal ultrasound and echocardiography as well as subsequently performed MRI and CT-scans led to the radiology-based diagnosis of WT of the right kidney with continuous vascular growth up into the right cardiac ventricle (Fig. 1). Interestingly, intravascular tumor extent appeared as a sacciforme/tubular and perfused structure, freely commuting back and forth over the tricuspid valve (check online ultrasound videos; for all online suppl. material, see www.karger.com/doi/10.1159/000496020). The patient was transferred to the oncological ward and treatment was initiated by four cycles of NAC with actinomycin D $45 \mu \mathrm{g} / \mathrm{kg}$ and vincristine $1.5 \mathrm{mg} / \mathrm{m}^{2}$, which quickly led to complete regression of upper venous congestion 
and tumor regression of about $75 \%$ (113 $\mathrm{mL}$ to $30 \mathrm{~mL})$ with nearly complete regression of previously solid intravascular tumor extensions. Intravascularly, only a membrane-like structure persisted. Alongside this treatment, prophylactic heparinization was carried out to avoid appositional thrombus development and prophylaxis of VOD (veno-occlusive disease) was achieved via ursodesoxycholic acid.

6 weeks after the initial diagnosis, the abdominothoracic tumor resection was performed in a multidisciplinary team. The procedure was started abdominally with complete nephrectomy. Since it was not possible to completely mobilize the cranial tumor extensions via abdominal cavotomy, the cardiothoracic surgeons continued with median sternotomy and application of cardiopulmonary bypass and deep hypothermia to acquire bloodless operation conditions. The intracardiac tumor extension could be completely resected, but intrahepatic VCI showed short-segment vessel-adhesive tumor elements with retrograde growth into the liver veins, which could not be securely resected completely nor repaired with a pericardial patch, which led to a grading of SIOP-stage III instead of stage I due to intravascular R2-resection (Fig. 2). The operation was completed by lymphadenectomy of hiliary, infra- and suprarenal lymph nodes. Histological assessment revealed a nephroblastoma of intermediary malignancy (mixed type) with about 75\% necrotic and 25\% vital areas. Lymph nodes were tumor-free. Postoperative recovery was speedy with extubation on the first and transfer to the oncological ward on the fifth postoperative day. Postoperative treatment according to SIOP2001 included adjuvant chemotherapy (AV2) and involved-field-radiation of VCI-walladhesive tumor residues with a cumulative dose of 25.2 Gray. The patient currently remains in remission and good health under ongoing adjuvant chemotherapy.

\section{Discussion}

The presented case highlights that even extreme vascular extent does not have to be synonymous with metastasized disease in WT, but can have a very favorable outcome, especially since most of intravascularly extending WTs exhibit the favorable intermediate risk pathology [1]. The presented case is particularly remarkable because of the uncommon morphology of the intravascular tumor extension as a sacciforme and perfused structure as well as the short segment retrograde growth into the liver veins and wall adhesion in that region. Despite of these features, there were no signs of primary metastasis and prognosis should be expected to be favorable [9]. Late complications such as radiation-associated atherosclerosis and thrombosis have been reported, but remain unlikely [10].

NAC has a special value in stabilizing such patients and in acquiring operability by downstaging and stabilizing tumor tissue. While there has been some debate between SIOP and NWTS (National Wilms tumor study group) investigators about advantages and disadvantages of NAC for WT $[11,12,13]$, patients with intravascular WT growth should clearly receive neoadjuvant treatment, since - just like our patient - about $50 \%$ exhibit downstaging of about $50 \%$ or more, some of them even subtotal or complete regression, leading to improved operability and reduced perioperative complications, such as tumor rupture or significant hemorrhage [1, 7]. Nonetheless, preoperative complications during NAC such as pulmonary embolism or ARDS (acute respiratory distress syndrome) can occur, but remain rarities [14]. Data on increased wall-adhesiveness due to NAC is very limited and incomplete thrombus resection has not been found to be an adverse prognostic factor in the NWTS-4 study [14].

During NAC, patients should be monitored closely and rare complications such as traumatic tumor rupture or Budd-Chiari-syndrome can require emergency surgery so that 
interdisciplinary discussion including involvement of surgeons should be held soon as possible after initial diagnosis of intravascular WT manifestation [15]. Preoperatively, ultrasound and echocardiography have a special value in evaluating wall-adhesiveness by depicting mobility of intravascular tumor extent and thereby helping in multidisciplinary operative planning. Even intraoperatively, TEE (trans-esophageal echocardiography) can be very helpful in evaluating vascular tumor extent [14].

Since most molecular analyses on WTs focus on correlation to malignancy and tumor progression $[16,17]$, little is known about tumor biology of vascular WT extensions. The not significantly worse outcome indicates that responsible molecular features are distinct from purely pro-invasive or pro-proliferative mutations. Analyses of WT study group cohorts regarding distinct molecular patterns favoring vascular extension could shed more light on the basis of this phenomenon.

\section{Statement of Ethics}

The authors have no ethical conflicts to disclose.

\section{Disclosure Statement}

The authors declare no conflict of interests.

\section{References}

1 Szavay P, Luithle T, Semler O, Graf N, Fuchs J. Surgery of cavoatrial tumor thrombus in nephroblastoma: a report of the SIOP/GPOH study. Pediatr Blood Cancer. 2004 Jul;43(1):40-5.

2 Abdullah Y, Karpelowsky J, Davidson A, Thomas J, Brooks A, Hewitson J, et al. Management of nine cases of Wilms' tumour with intracardiac extension - a single centre experience. J Pediatr Surg. 2013 Feb;48(2):3949.

3 Cox SG, Davidson A, Thomas J, et al. Surgical management and outcomes of 12 cases of Wilms tumour with intracardiac extension from a single centre. Pediatr Surg Int. 2018 Feb;34(2):227-235.

4 Erginel B, Ugurlucan M, Basaran M, Buget M, Yuksel S, Celik A, et al. Management of a Wilms' tumor with intracardiac extension using extracorporeal circulation and deep hypothermic circulatory arrest: case report and review of the literature. Pediatr Hematol Oncol. 2016 Feb;33(1):67-73.

5 Namboodiri N, Krishnamoorthy KM, Tharakan JA. Intra-atrial extension of Wilms' tumor. J Am Soc Echocardiogr. 2008 Jan;21(1):91.e3-4.

6 Ehrlich PF. Bilateral Wilms' tumor: the need to improve outcomes. Expert Rev Anticancer Ther. 2009 Jul;9(7):963-73.

7 Spreafico F, Bellani FF. Wilms' tumor: past, present and (possibly) future. Expert Rev Anticancer Ther. 2006 Feb;6(2):249-58.

8 Daum R, Roth H, Zachariou Z. Tumor infiltration of the vena cava in nephroblastoma. Eur J Pediatr Surg. 1994 Feb;4(1):16-20.

9 Fuchs J. [Surgical concepts in the treatment of Wilms tumor: an update]. Urologe A. 2015 Dec;54(12):178491.

10 Gilbert JD, Byard RW. Fatal ischemic enteritis with hemorrhage-a late complication of treated Wilms tumor. J Forensic Sci. 2013 Jan;58(1):234-6.

11 Bhatnagar S, Bhatnagar S. Management of Wilms' tumor: NWTS vs SIOP. J Indian Assoc Pediatr Surg. 2009 Jan;14(1):6-14.

12 Schenk JP, Graf N, Günther P, Ley S, Göppl M, Kulozik A, et al. Role of MRI in the management of patients with nephroblastoma. Eur Radiol. 2008 Apr;18(4):683-91.

13 Brisse HJ, Smets AM, Kaste SC, Owens CM. Imaging in unilateral Wilms tumour. Pediatr Radiol. 2008 Jan;38(1):18-29. 
Imle et al.: A Severe Case of Intracardiac Wilms Tumor Extension

14 Khozeimeh N, Sinha P, Dome JS, Guzzetta PC Jr. Strategy for management of retroperitoneal tumors with caval tumor thrombus. J Pediatr Surg. 2011 Nov;46(11):2065-70.

15 McMahon S, Carachi R. Wilms' tumor with intravascular extension: A review article. J Indian Assoc Pediatr Surg. 2014 Oct;19(4):195-200.

16 Zirn B, Hartmann O, Samans B, Krause M, Wittmann S, Mertens F, et al. Expression profiling of Wilms tumors reveals new candidate genes for different clinical parameters. Int J Cancer. 2006 Apr;118(8):1954-62.

17 Scott RH, Murray A, Baskcomb L, et al. Stratification of Wilms tumor by genetic and epigenetic analysis. Oncotarget. 2012;3(3):1-9.

A

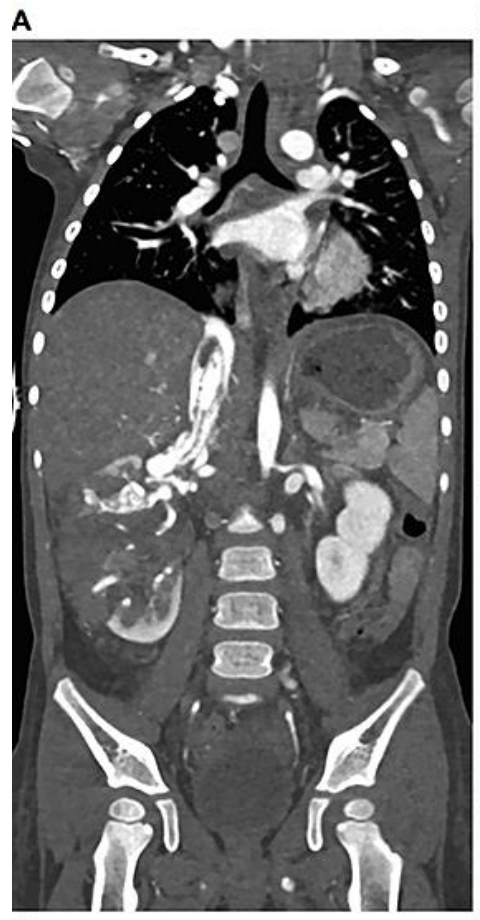

D

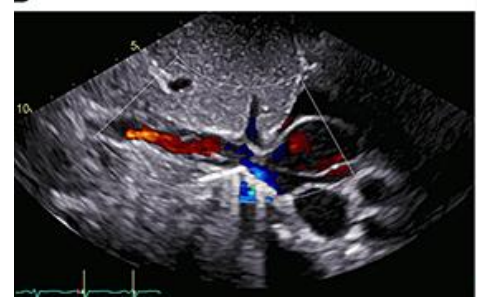

B

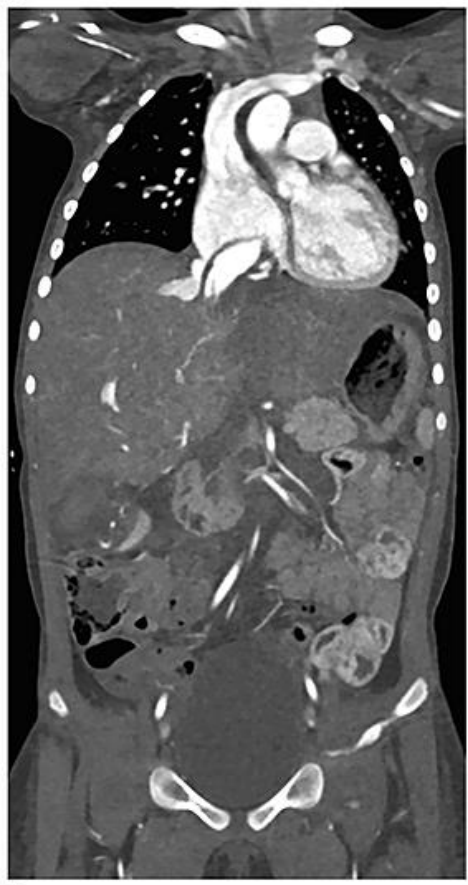

E

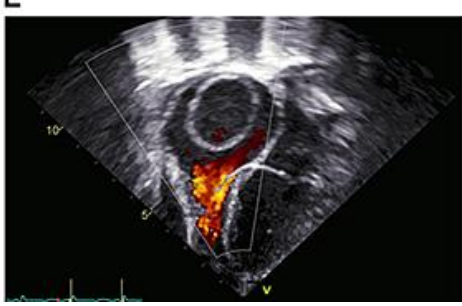

C

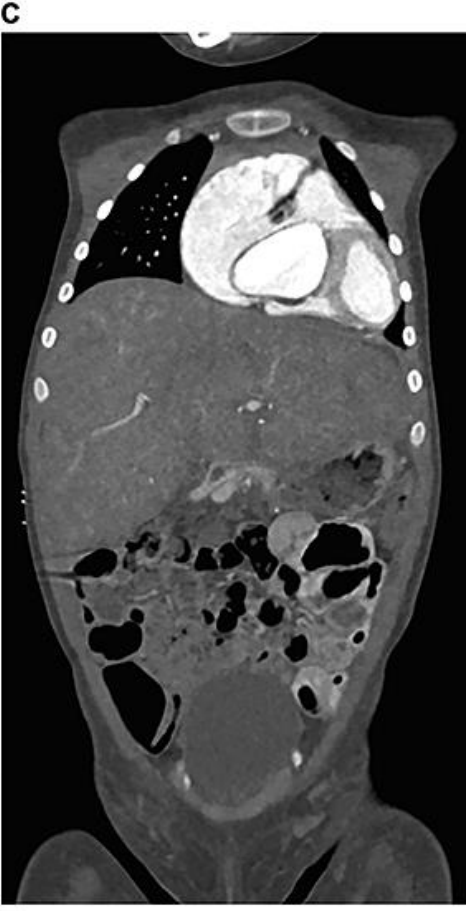

F

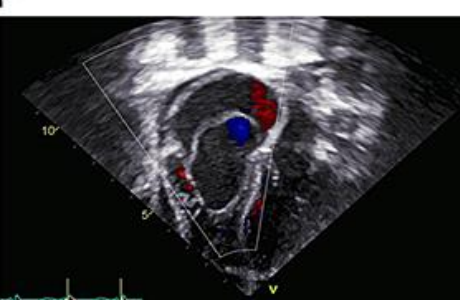

Fig. 1. A-C. Coronal CT reconstruction in early venous phase after i.v. contrast application, depicting primary tumor and vascular extent. D-F. Ultrasound images in Doppler mode highlighting hepatoatrial transition (D) as well as atrial dilation and movement of the sacciforme intravascular tumor extension over the tricuspid valve into the right cardiac ventricle ( $E$ and $\mathbf{F}$ ). All depicted images represent the initial diagnosis. 

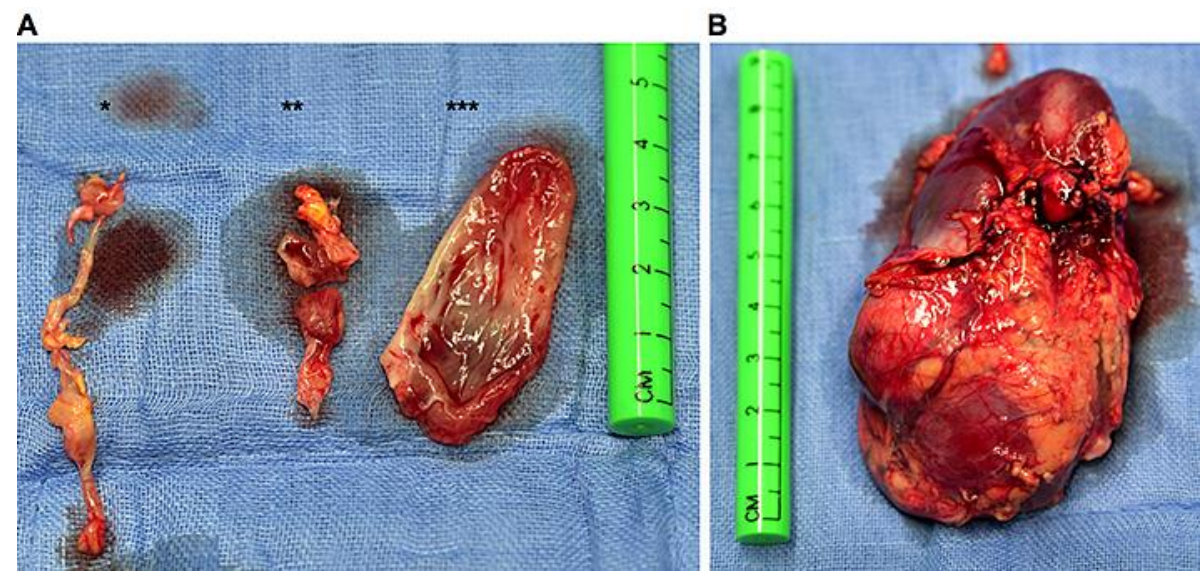

Fig. 2. A. Intravascular tumor extensions along centimeter scale: * subhepatic VCI; ** intrahepatic VCI; *** intracardiac. B. Dissected kidney along centimeter scale. 